\title{
Tabersonine ameliorates osteoblast apoptosis in rats with dexamethasone-induced osteoporosis by regulating the Nrf2/ROS/Bax signalling pathway
}

\author{
Xi Sun ${ }^{1}$, Lijun Gan ${ }^{1}$, Nan Li ${ }^{1}$, Shuyi Sun ${ }^{2}$ and Na Li ${ }^{1^{*}}$
}

\begin{abstract}
We explored how tabersonine (Tab) protected against dexamethasone (Dex)-induced osteoporosis. Osteoblasts were treated with Dex $(100 \mu \mathrm{M})$ with or without Table $(5$ or $10 \mu \mathrm{M})$. We measured cell viability, alkaline phosphatase (ALP) activity, and mitochondrial superoxide and reactive oxygen species levels. We used flow cytometry to explore the effects of Tab on mitochondrial membrane potential and osteoblast apoptosis. We used RT-PCR and western blotting to examine the effect of Tab on protein expression. We evaluated the effects of Tab on bone histopathology and bone mineral density in rats with Dex-induced osteoporosis. Tab increased cell viability and ALP activity, and reduced the mitochondrial superoxide, reactive oxygen species and matrix metalloproteinase levels and osteoblast apoptosis. Tab significantly reduced the levels of nuclear factor erythroid 2-related factor 2 (Nrf2), haem oxygenase-1 and NAD(P) $\mathrm{H}$ quinone dehydrogenase 1. Moreover, it increased the levels of mRNAs encoding runt-related transcription factor 2 , bone morphogenetic protein-2 and osterix. These data suggest that Tab ameliorates Dex-induced osteoporosis by regulating the Nrf2 signalling pathway.
\end{abstract}

Keywords: Tabersonine, Dexamethasone, Osteoporosis, Osteoblast, Inflammation, Oxidative stress

\section{Introduction}

Osteoporosis is a metabolic bone disease characterised by the loss of bone mass and bone weakness; it affects about 200 million individuals $(80 \%$ of whom are female) worldwide (Sözen et al. 2017). Skeletal integrity is compromised in osteoporotic patients; an imbalance develops between bone formation and resorption, triggering fracture (Office of the Surgeon General (USA) 2004). Osteoblasts are responsible for bone formation and mineralisation. Several factors regulate osteoblast activity. Oestrogen enhances osteoblastic mineralisation and stimulates osteoblast differentiation and proliferation (Domazetovic et al. 2017). In females, osteoporosis

\footnotetext{
*Correspondence: 414110808@qq.com

${ }^{1}$ Department of Neonatology, First Hospital of Jilin University, No. 71 Xinmin Street, Chaoyang District, Changchun 130021, Jilin, China Full list of author information is available at the end of the article
}

commonly develops after menopause or ovariectomy (Luo et al. 2014). Nuclear factor erythroid 2-related factor 2 (Nrf2) expression contributes to osteoporosis development by enhancing reactive oxygen species (ROS) production (Ma et al. 2013). Mitochondrial dysfunction enhances osteocyte apoptosis, reducing bone mineralisation via ROS overproduction (Smietana et al. 2010). The antioxidant responsive element, in combination with the Nrf2 activator, prevents osteoporosis development by enhancing the expression levels of antioxidant enzymes (Jin et al. 2020). Osteoblast activity is regulated by the Nrf2 pathway (Sun et al. 2015). Conventional osteoporosis treatments have several limitations; alternative medicines have shown promise over the past few decades.

Tabersonine (Tab), originally isolated from Catharanthus roseus (Apocynaceae), is a terpene indole alkaloid found in several medicinal plants (Almagro et al. 2015). 
C. roseus is traditionally used in China for the management of cancer, malaria and diabetes (Nejat et al. 2015). Tab has strong anticancer and neuroprotective effects (Nejat et al. 2015). It protects against lung injury by regulating the NF- $\mathrm{kB}$ and $\mathrm{p} 38 / \mathrm{MK} 2$ pathways, thereby strongly inhibiting inflammation (Zhang et al. 2018). In this study, we explored whether Tab protected against dexamethasone (Dex)-induced osteoporosis, and the molecular mechanism in play.

\section{Materials and methods}

\section{Animals}

Neonatal Sprague-Dawley rats (strain P2) were sacrificed via decapitation and their calvaria were isolated for in vitro analysis. The in vivo study was performed with 8 -week-old female rats (mean body weight $250 \pm 25 \mathrm{~g}$ ), held under standard laboratory conditions. All animal procedures were approved by the animal ethics committee of the First Hospital of Jilin University, China (Approval no. IAEC/FH-JU/2018/01).

\section{Chemicals}

Tab was supplied by the Institute of Botany, Chinese Academy of Sciences, China; Dex was obtained from Tianjin Lisheng Pharmaceutical Co. Ltd., China. Alkaline phosphatase (ALP), Nrf2, and matrix metalloproteinase detection kits were purchased from Abcam, USA. The antibodies used for western blotting and the qRT-PCR primers were obtained from Thermo Fisher Scientific, USA.

\section{Osteoblast isolation and culture}

Calvarial osteoblasts were isolated from neonatal rats as described previously (Liu et al. 2019). The connective tissue was cleaned carefully and digested with $0.3 \%$ $(\mathrm{w} / \mathrm{v})$ type II collagenase after isolation of the parietal bones. The cells were cultured in DMEM supplemented with antibiotics and antimycotics, charcoal dextrantreated foetal blood serum and non-essential amino acids $(100 \mu \mathrm{M})$ for $48 \mathrm{~h}$.

\section{Estimation of cell viability}

Cell proliferation was estimated using the CCK- 8 assay as described previously (Jo et al. 2015). Cells $\left(2 \times 10^{4} /\right.$ well) were seeded into six-well plates, incubated for $24 \mathrm{~h}$, treated with $100 \mu \mathrm{M}$ Dex with or without the addition of Table $(5$ or $10 \mu \mathrm{M}) 2 \mathrm{~h}$ previously, and cultured for $24 \mathrm{~h}$; CCK-8 was then added, followed by incubation for $2 \mathrm{~h}$. Cell viability was determined by estimating absorbance at $450 \mathrm{~nm}$ using a microplate reader.

\section{Measurement of ALP activity}

Osteoblasts $\left(2 \times 10^{5}\right.$ cells/well $)$ were seeded into sixwell plates and treated with Dex with or without Tab for various durations. The cells were lysed with lysis buffer, and ALP levels were measured as directed by the kit manufacturer.

\section{Mitochondrial superoxide and ROS assays}

Flow cytometry was used to measure mitochondrial superoxide and ROS levels in osteoblasts, with the use of the MitoSOXTM Red indicator and a nonfluorescent 2,7-dichlorofluorescin diacetate probe, respectively.

\section{Nrf2 assay}

The TransAM Nrf2 assay was used to estimate Nrf2 activity, according to the manufacturer's instructions. An ELX800 microplate reader was employed to measure absorbance at $405 \mathrm{~nm}$.

\section{Mitochondrial membrane potential}

Mitochondrial membrane potential (MMP) was estimated using the lipophilic, cationic fluorescent dye $5,5^{\prime}, 6,6^{\prime}$-tetrachloro- $1,1^{\prime}, 3,3^{\prime}$-tetraethylbenzimidazolcarbocyanine iodide. Osteoblasts $\left(2 \times 10^{5}\right.$ cells/well $)$ were seeded into six-well plates and the Mitochondrial Membrane Potential Detection Kit was used to estimate MMP according to the manufacturer's instructions.

\section{Osteoblast apoptosis}

Osteoblasts $\left(2 \times 10^{5}\right.$ cells/well $)$ were seeded into sixwell plates and treated with Dex with or without Tab for various durations. The cells were resuspended in $500 \mu \mathrm{l}$ binding buffer containing PI $(5 \mu \mathrm{l})$ and Annexin V-APC $(5 \mu \mathrm{l})$, rinsed with PBS, and placed on ice. Flow cytometry was used to measure apoptosis.

\section{qRT-PCR}

Osteoblasts were cultured with Tab for $48 \mathrm{~h}$. Total RNA was extracted using the TRIZOL reagent and cDNA was synthesised using SuperScript II reverse transcriptase. qRT-PCR was performed using the SYBR Green PCR Master Mix system. GAPDH served as the internal control. Cycle number (the Cq value) was plotted against the log of the added cDNA concentration, and relative target gene expression levels were determined using the $2^{-\Delta \Delta C q}$ method. 


\begin{tabular}{lll}
\hline BMP-2 & Forward & 5'-TGCGGTCTCCTAAAGGTCG-3' \\
& Reverse & 5'-ACTCAAACTCGCTGAGGACG-3' \\
Runx2 & Forward & 5'-TCTCCAGGAGGACAGCAAGG-3' \\
& Reverse & 5'-TTGCAGCCTTAAATGACTCGGT-3' \\
Osterix & Forward & 5'-CACTCTCCCTGCCAGACCTC-3' \\
& Reverse & 5'-GCCATAGTGAACTTCCTCCTCAAG-3' \\
3-actin & Forward & 5'-GGAGATTACTGCCCTGGCTCCTA-3' \\
& Reverse & 5'-GACTCATCGTACTCCTGCTTGCTG-3' \\
\hline
\end{tabular}

\section{Western blotting}

Total osteoblast proteins were extracted into ice-cold radioimmunoprecipitation lysis buffer, and the DC Protein Assay was performed to estimate protein levels. Sodium dodecyl sulphate-polyacrylamide gel $(10 \%$ $\mathrm{w} / \mathrm{v}$ ) electrophoresis was used to separate the proteins, which were then transferred to polyvinylidene difluoride membranes. The membranes were incubated with a 5\% $(w / v)$ fresh nonfat dry milk solution to block non-specific reactions. They were incubated at $4{ }^{\circ} \mathrm{C}$ overnight with primary antibodies against Nrf2 (1:500), Ho-1 (1:1000), NAD $(\mathrm{P}) \mathrm{H}$ quinone dehydrogenase 1 (NQO-1; $1: 1000)$, caspase-3 (1:500), Bcl-2 (1:1000), Bax (1:1000), cytochrome- $C$ (1:200) and $\beta$-actin (1:1000), and then at room temperature for $60 \mathrm{~min}$ with appropriate secondary antibodies. The enhanced chemiluminescence assay was used to detect protein bands, and the ImageLab software was employed for densitometric analysis.

\section{Osteoporosis induction}

Rats were separated into four groups: control, Dex (1 mg/ $\mathrm{kg}$ intramuscularly, daily for 60 days), and Tables 20 and 40 (20 and $40 \mathrm{mg} / \mathrm{kg}$ intraperitoneally, $30 \mathrm{~min}$ after Dex administration for 60 days). The animals were then sacrificed, and the bilateral femora were collected.

\section{Determination of bone mineral density}

Dual energy X-ray absorptiometry was used to determine the bone mineral density (BMD). Rats were anesthetised with Zoletil/Rompun, and the femora, lumbar vertebrae and whole body were scanned for the evaluation of BMD.

\section{Histopathological analysis}

The isolated tibiae were fixed in $10 \%(\mathrm{v} / \mathrm{v})$ formalin at $40{ }^{\circ} \mathrm{C}$ for 2 days. Ethylenediaminetetraacetic acid $(10 \%$ $\mathrm{v} / \mathrm{v}$ ) was used to decalcify the bones, which were then placed in liquid paraffin to create wax blocks. Sections ( $4 \mu \mathrm{m}$ thickness) were prepared and stained with haematoxylin and eosin. Histopathological changes were identified under a tri-ocular microscope at $100 \times$ magnification.

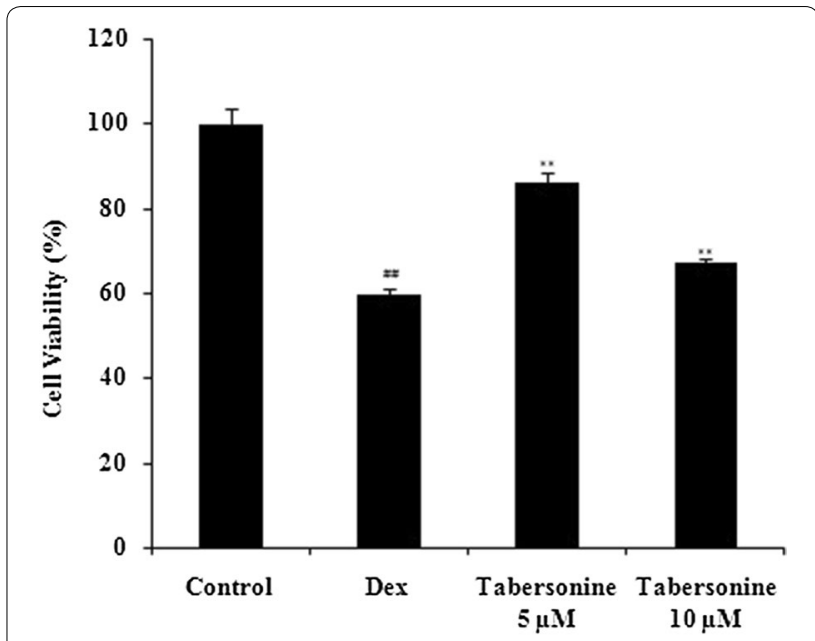

Fig. 1 Effect of tabersonine on osteoblast viability after dexamethasone treatment, evaluated using the CCK-8 assay. Means \pm SEMs $(n=10) .{ }^{\# \#} p<0.01$ vs. control, ${ }^{* *} p<0.01$ vs. Dex group

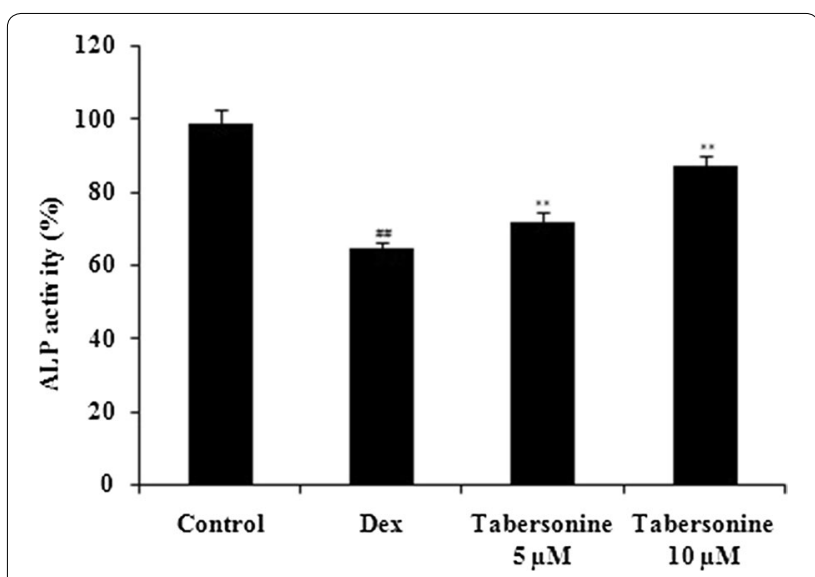

Fig. 2 Effect of tabersonine on osteoblast alkaline phosphatase activity. Means \pm SEMs $(n=10) .{ }^{\# \#} p<0.01$ vs. control, ${ }^{* *} p<0.01$ vs. Dex group

\section{Statistical analysis}

All data are expressed as means \pm standard errors of the means ( $n=10$ /group). One-way analysis of variance, followed by the Dunnett test, was performed using the GraphPad Prism software (ver. 6.1; GraphPad Software Inc., USA). $p$ values $<0.05$ were considered to reflect statistical significance. 


\section{Results}

\section{Effect of Tab on osteoclast viability}

Dex reduced osteoblast viability (compared with the control) and Tab rescued viability, as revealed by the CCK-8 assay (Fig. 1).

\section{Effect of Tab on ALP activity}

Dex significantly reduced the osteoblast ALP level compared with the control ( $p<0.01$; Fig. 2$)$, and Tab neutralised this negative effect of Dex.
Effects of Tab on mitochondrial superoxide and ROS levels The effects of Tab on mitochondrial superoxide and ROS levels, as determined by flow cytometry, are shown in Fig. 3. Dex enhanced the production of mitochondrial superoxide and ROS compared with the control. Tab neutralised these negative effects of Dex.

\section{Effect of Tab on the MMP}

The MMP was measured by estimating mitochondrial depolarisation via the change in the red/green fluorescence ratio (Fig. 4). The MMP was lesser in Dex-treated osteoblasts than in controls, and Tab neutralised this negative effect.
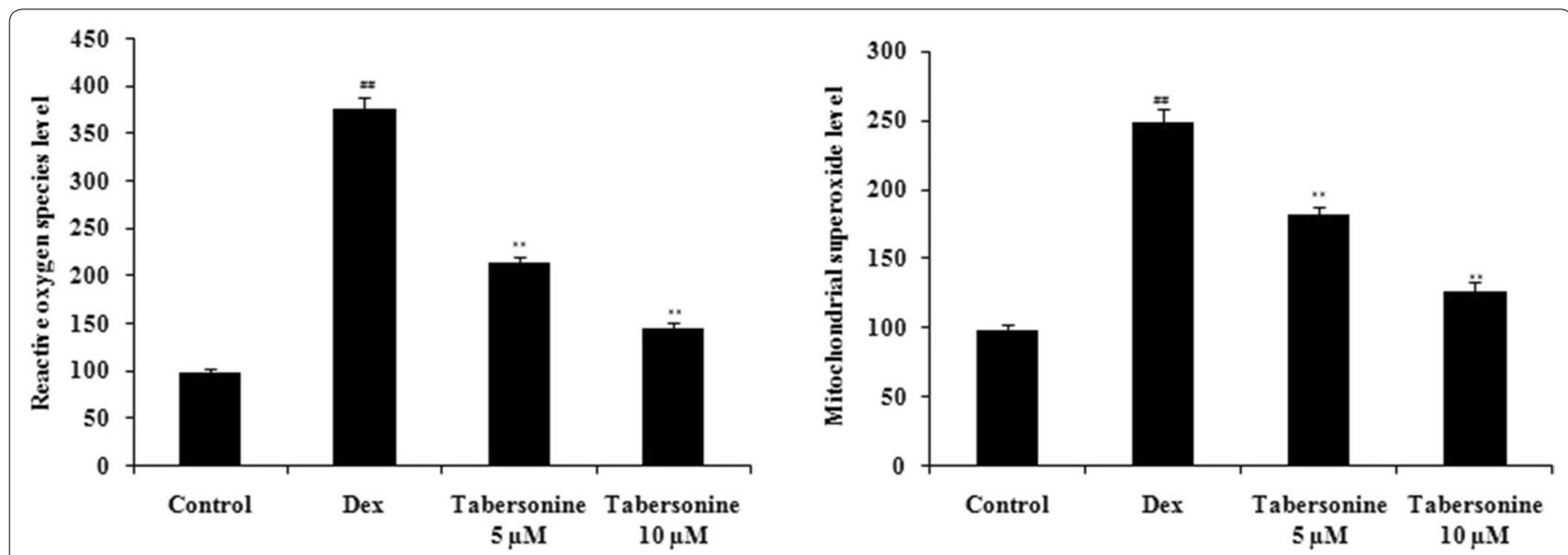

Fig. 3 Effect of tabersonine on mitochondrial superoxide and reactive oxygen species production in osteoblasts, measured via flow cytometry. Means \pm SEMs $(n=10) . \# p<0.01$ vs. control, ${ }^{* *} p<0.01$ vs. Dex group
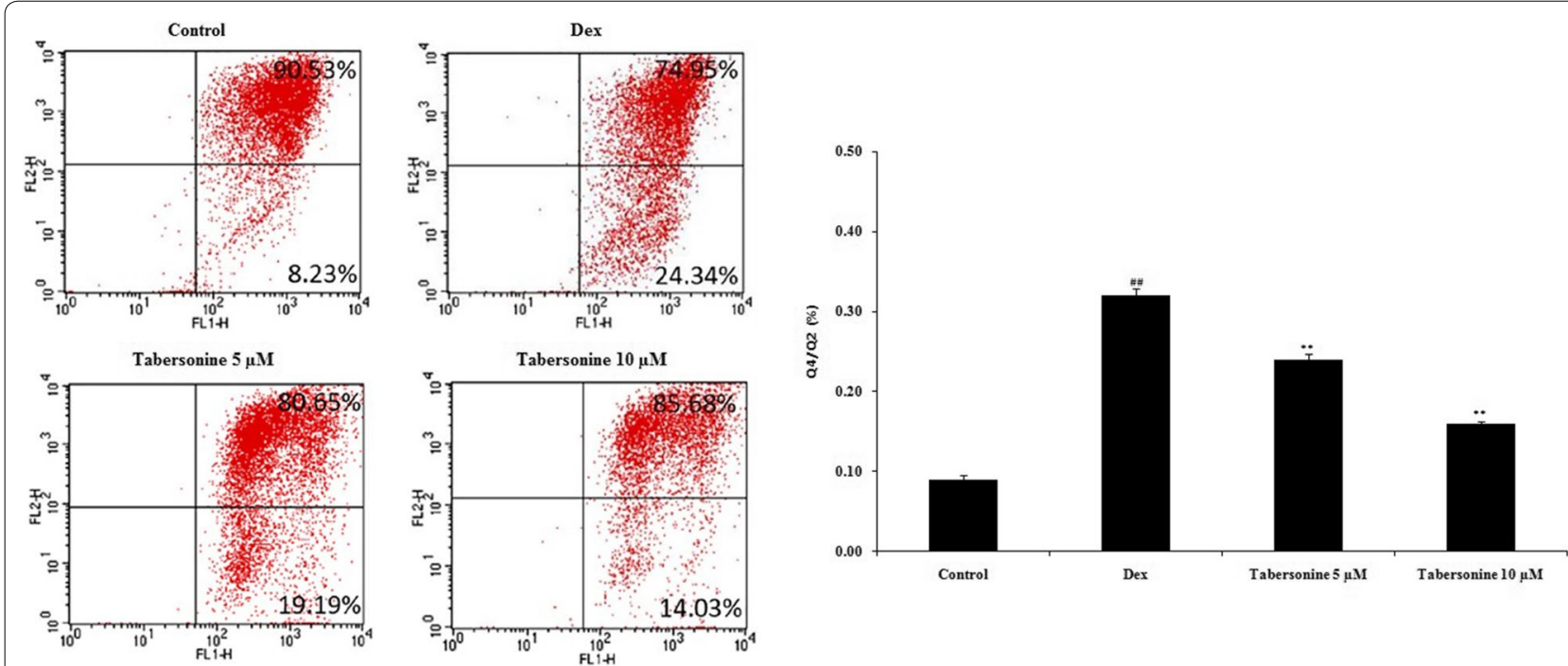

Fig. 4 Effect of tabersonine on the mitochondrial membrane potential of osteoblasts treated with dexamethasone. Means $\pm S E M s$ $(n=10) .{ }^{\#} p<0.01$ vs. control, ${ }^{* *} p<0.01$ vs. Dex group 


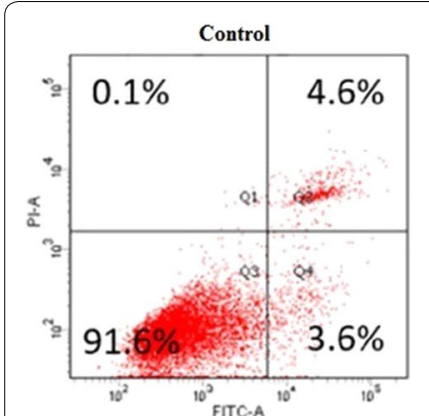

Tabersonine $5 \mu \mathrm{M}$

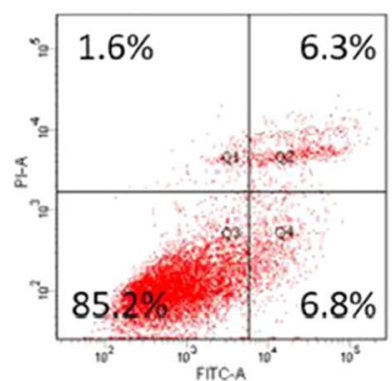

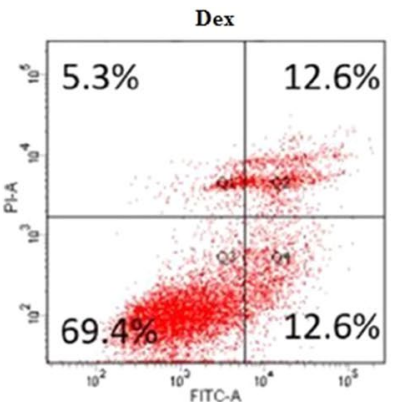

Tabersonine $10 \mu \mathrm{M}$

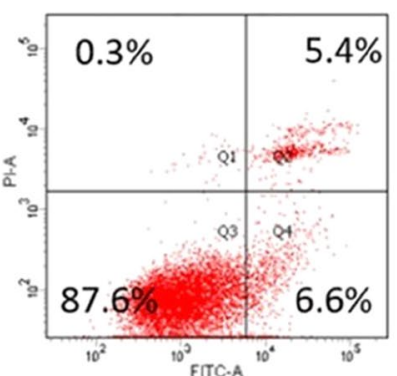

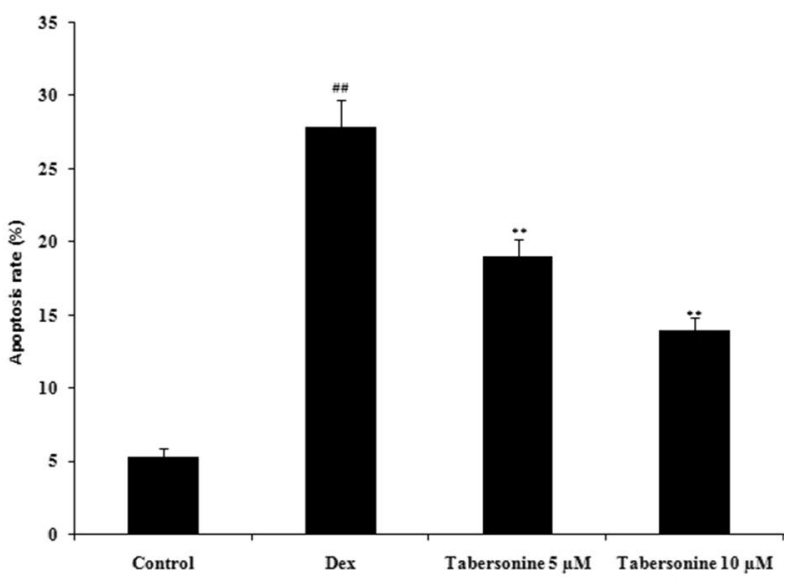

Fig. 5 Effect of tabersonine on apoptosis of dexamethasone-treated osteoblasts. Means \pm SEMs $(n=10) . \# p<0.01$ vs. control, ${ }^{* *} p<0.01$ vs. Dex group

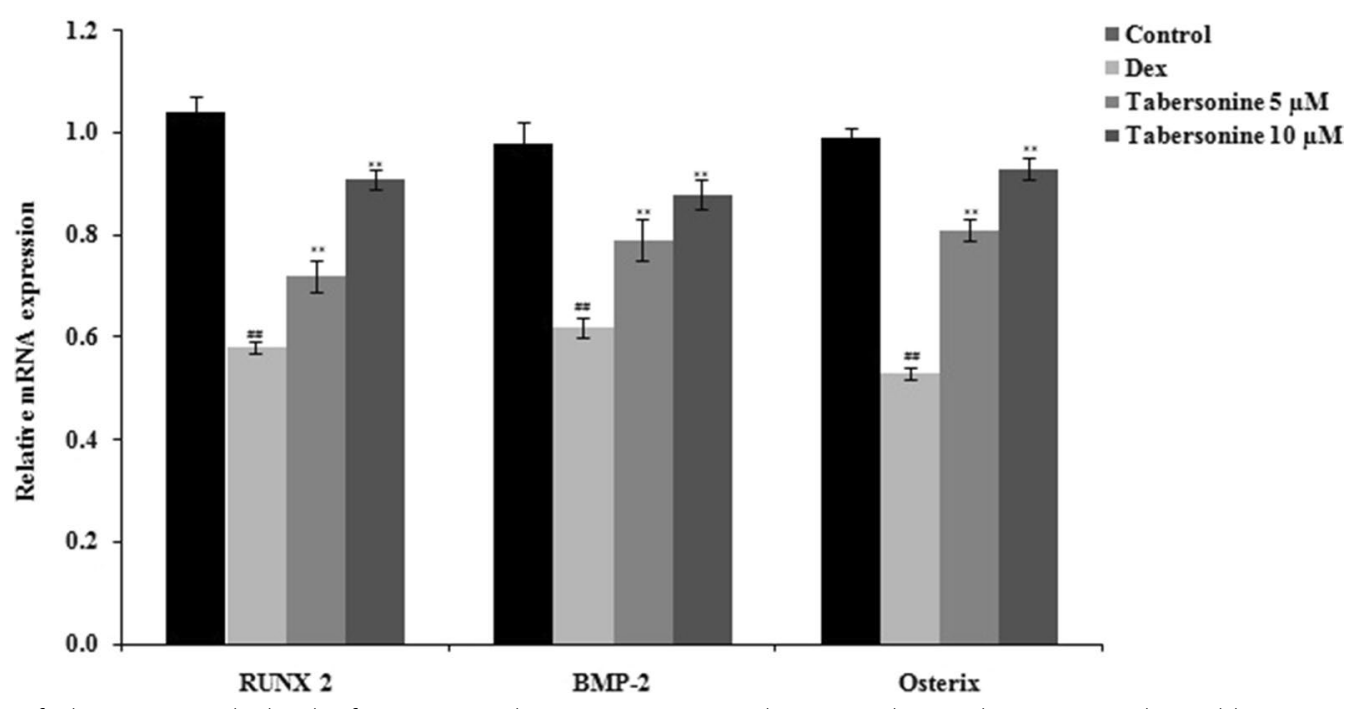

Fig. 6 Effects of tabersonine on the levels of mRNAs encoding RUNX2, BMP-2 and osterix in dexamethasone-treated osteoblasts. Means \pm SEMs $(n=10) .{ }^{\# \#} p<0.01$ vs. control, ${ }^{* *} p<0.01$ vs. Dex group

\section{Effect of Tab on osteoblast apoptosis}

Osteoblast apoptosis was measured via flow cytometry (Fig. 5). Apoptosis was greater in Dex-treated than in control osteoblasts, and Tab neutralised this negative effect of Dex.
Effects of Tab on the levels of mRNAs encoding runt-related transcription factor 2 , bone morphogenetic protein-2 and osterix

The levels of mRNAs encoding runt-related transcription factor 2 (RUNX2), bone morphogenetic protein-2 


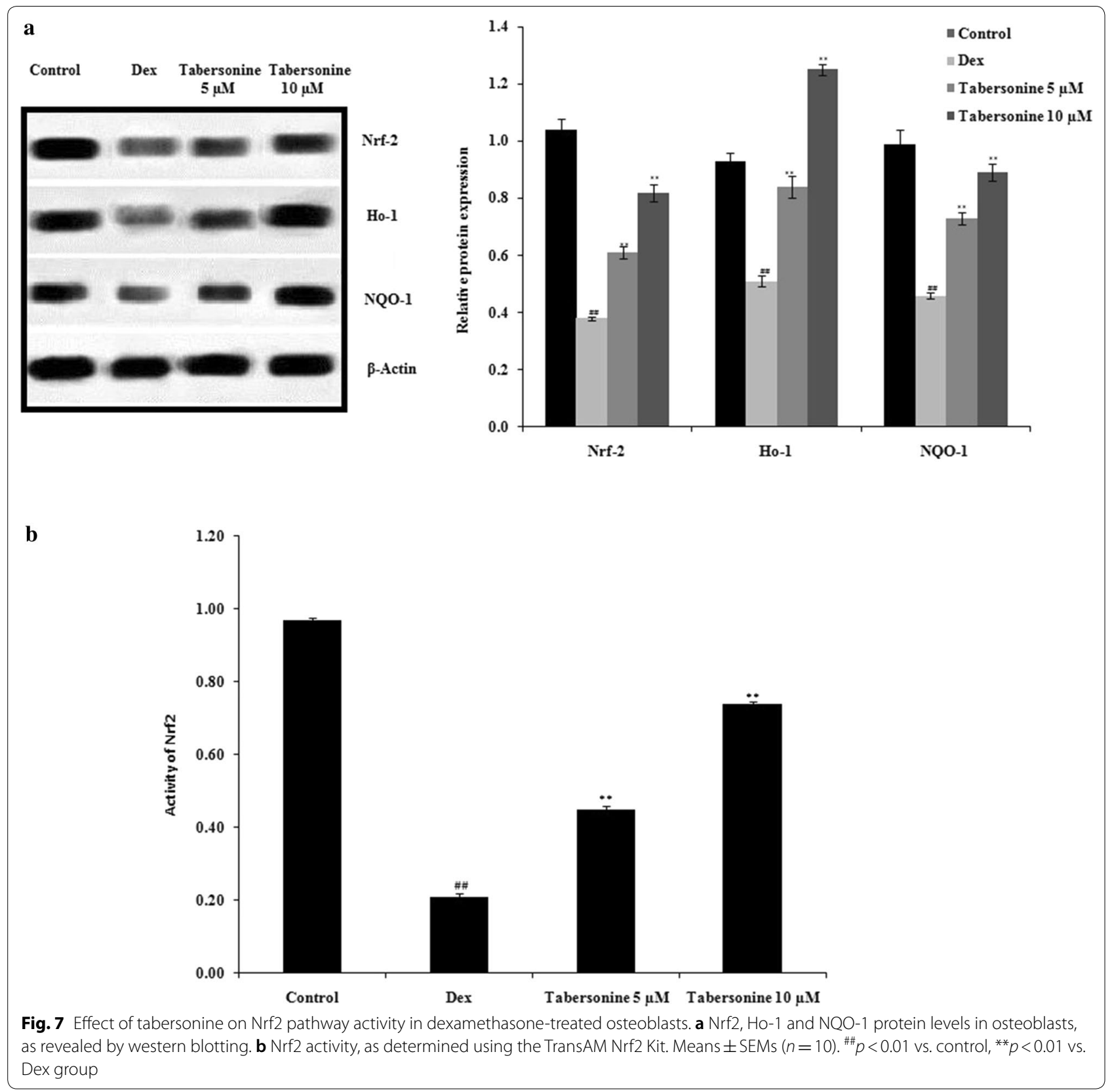

(BMP-2) and osterix were measured in osteoblasts treated with Dex alone or Dex and Tab (Fig. 6). The mRNA levels decreased upon Dex treatment, and Tab neutralised these negative Dex effects.

\section{Effect of Tab on Nrf2 signalling}

The effect of Tab on Nrf2 signalling was evaluated by measuring the levels of Nrf2, Ho-1 and NQO-1 in osteoblasts via western blotting; Nrf2 activity was also determined using the TransAM Nrf2 kit (Fig. 7a, b). The Nrf2, Ho-1 and NQO-1 levels were lower in Dex-treated than in control osteoblasts. Tab significantly neutralised the negative effects of Dex (Fig. 7a). Nrf2 activity was reduced in Dex-treated cells compared with control cells. Again, Tab neutralised this negative effect of Dex (Fig. 7b).

Effects of Tab on the expression of caspase-3, Bcl-2, Bax and cytochrome- $C$

Tab affected the levels of pre- and pro-apoptotic proteins in Dex-treated osteoblasts (Fig. 8). Dex significantly increased the levels of caspase-3, Bax and 

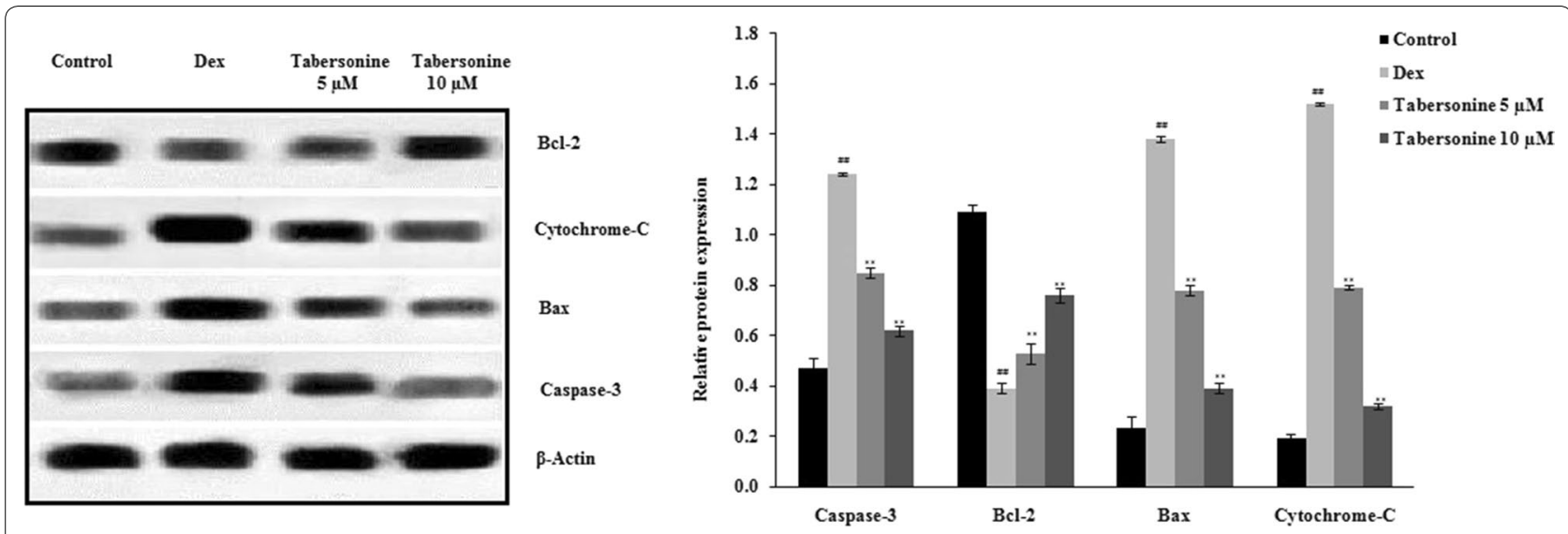

Fig. 8 Effects of tabersonine on the levels of caspase-3, Bcl-2, Bax and cytochrome-C in osteoblasts. Means \pm SEMs $(n=10)$. ${ }^{\# \#} p<0.01$ vs. control, ${ }^{* *} p<0.01$ vs. Dex group
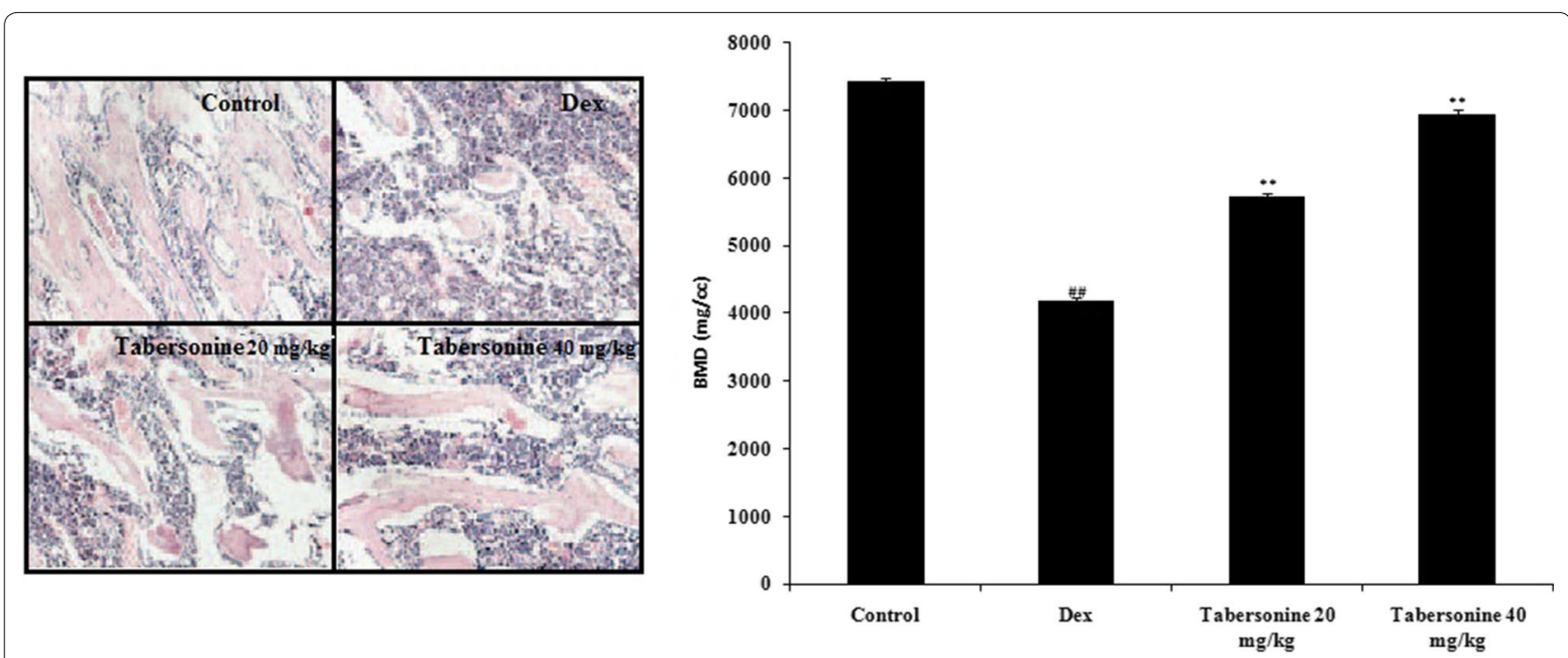

Fig. 9 Tabersonine ameliorated Dex-induced osteoporosis in rats. a Histopathological bone changes revealed by H\&E staining (100x). b BMD in rats with Dex-induced osteoporosis treated or not treated with Tab. Means \pm SEMs $(n=10) .{ }^{\# \#} p<0.01$ vs. control, ${ }^{* *} p<0.01$ vs. Dex group

cytochrome-c and reduced that of Bcl-2 compared with controls. Tab neutralised these adverse effects of Dex.

\section{Effects of Tab on bone histopathology and BMD}

Tab affected bone histopathology and BMD in rats with Dex-induced osteoporosis (Fig. 9). The control group exhibited normal bone. Dex destroyed bone trabeculae (as revealed by staining) and reduced bone synthesis. Tab neutralised these negative effects. The BMD was significantly lower in the Dex group than in the control group; Tab largely neutralised this deterioration.

\section{Discussion}

Bone homeostasis is affected by osteoporosis, a disease characterised by the dysregulation of bone formation and resorption. Osteoblasts regulate bone formation and osteoclasts control bone resorption (Feng and McDonald 2011). Dex compromises osteoblast function; Dex-induced models of osteoporosis are used widely for the pharmacological evaluation of potentially useful anti-osteoporosis drugs (Yin et al. 2019). We employed a Dex-induced osteoporosis model to explore the effects of Tab on osteoblast viability, ALP activity, mitochondrial superoxide and ROS production, the MMP and apoptosis (the latter two parameters were assessed by flow cytometry). We performed 
RT-PCR and western blotting to explore the effects of Tab on osteoblast protein expression.

Mitochondria play important roles in cell survival and apoptosis (Wang and Youle 2009). Dex affects mitochondrial respiration by inducing ROS synthesis, in turn activating the cytochrome- $C$-associated pro-apoptotic pathway and the caspase cascade (Liu et al. 2018). Tab treatment enhanced the viability of Dex-treated osteoblasts and ALP activity, and reduced mitochondrial superoxide and ROS production. Tab attenuated the expression of caspase-3, Bcl-2, Bax, and cytochrome$c$ in Dex-treated osteoblasts. Flow cytometric analysis revealed that Tab enhanced the MMP and reduced apoptosis of Dex-treated osteoblasts.

Nrf2 signalling protects osteoblasts (Sena and Chandel 2012). The mitochondrial ROS level is regulated by Ho-1, NQO-1, and Nrf2 (Li et al. 2014). In models of osteoporosis, the Nrf2 pathway is suppressed, accompanied by mitochondrial dysfunction (Huang et al. 2019). We observed higher levels of Nrf2, Ho-1 and NQO-1 in the Tab groups than in the Dex group. The levels of mRNAs encoding RUNX2, BMP-2 and osterix were significantly enhanced in the Tab-treated groups compared with the Dex-treated group (all $p<0.01$ ). In conclusion, our findings suggest that Tab ameliorates Dex-induced osteoporosis by regulating $\mathrm{Nrf} 2 / \mathrm{ROS} / \mathrm{Bax}$ signalling. Thus, Tab may be clinically valuable for osteoporosis management.

\section{Acknowledgements}

The authors thank the First Hospital of Jilin University, China, for providing facilities.

\section{Authors' contributions}

XS and LG performed the experiments and collected data. NL was involved in the statistical analysis. SS evaluated the histopathological data. NL designed and supervised the work and drafted the manuscript, although all authors contributed to manuscript preparation. All authors read and approved the final manuscript.

\section{Funding}

No external funding was received.

\section{Availability of data and materials}

The supporting data are not available for ethical reasons.

\section{Ethics approval and consent to participate}

The animal experiments were approved by the animal ethics committee of the First Hospital of Jilin University, China (No. IAEC/FH-JU/2018/01).

\section{Consent for publication}

Not applicable.

\section{Competing interests}

No author has any possibly competing interests.

\section{Author details}

${ }^{1}$ Department of Neonatology, First Hospital of Jilin University, No. 71 Xinmin Street, Chaoyang District, Changchun 130021, Jilin, China. ${ }^{2}$ Department of Ultrasonography, Jilin Provincial People's Hospital, Changchun 130000, Jilin, China.
Received: 23 May 2020 Accepted: 27 August 2020

Published online: 11 September 2020

\section{References}

Almagro L, Fernández-Pérez F, Pedreño MA (2015) Indole alkaloids from Catharanthus roseus: bioproduction and their effect on human health. Molecules 20(2):2973-3000

Feng X, McDonald JM (2011) Disorders of bone remodeling. Annu Rev Pathol 6:121-145

Huang MLH, Chiang S, Kalinowski DS, Bae DH, Sahni S, Richardson DR (2019) The role of the antioxidant response in mitochondrial dysfunction in degenerative diseases: cross-talk between antioxidant defense autophagy, and apoptosis. Oxid Med Cell Longev 2019:6392763

Jin W, Zhu X, Yao F, Xu X, Chen X, Luo Z, Zhao D, Li X, Leng X, Sun L (2020) Cytoprotective effect of Fufang Lurong Jiangu capsule against hydrogen peroxide-induced oxidative stress in bone marrow stromal cell-derived osteoblasts through the Nrf2/HO-1 signaling pathway. Biomed Pharmacother 121:109676

Jo HY, Kim Y, Park HW, Moon HE, Bae S, Kim J, Kim DG, Paek SH (2015) The unreliability of MTT assay in the cytotoxic test of primary cultured glioblastoma cells. Exp Neurobiol 24(3):235-245

Li L, Dong H, Song E, Xu X, Liu L, Song Y (2014) Nrf2/ARE pathway activation, HO-1 and NQO1 induction by polychlorinated biphenyl quinone is associated with reactive oxygen species and PI3K/AKT signaling. Chem Biol Interact 209:56-67

Liu S, Zhou L, Yang L, Mu S, Fang T, Fu Q (2018) Gastrodin alleviates glucocorticoid induced osteoporosis in rats via activating the Nrf2 signaling pathways. Oncotarget 9(14):11528-11540

Liu B, Lu Y, Wang Y, Ge L, Zhai N, Han J (2019) A protocol for isolation and identification and comparative characterization of primary osteoblasts from mouse and rat calvaria. Cell Tissue Bank 20(2):173-182

Luo K, Ma S, Guo J, Huang Y, Yan F, Xiao Y (2014) Association between postmenopausal osteoporosis and experimental periodontitis. Biomed Res Int. 2014:316134

Ma Q (2013) Role of nrf2 in oxidative stress and toxicity. Annu Rev Pharmacol Toxicol 53:401-426

Nejat N, Valdiani A, Cahill D, Tan YH, Maziah M, Abiri R (2015) Ornamental exterior versus therapeutic interior of Madagascar periwinkle (Catharanthus roseus): the two faces of a versatile herb. Sci World J 2015:982412

Office of the Surgeon General (US) (2004) Bone health and osteoporosis: a report of the surgeon general. 2, The basics of bone in health and disease. Office of the Surgeon General (US), Rockville

Sena LA, Chandel NS (2012) Physiological roles of mitochondrial reactive oxygen species. Mol Cell 48(2):158-167

Smietana MJ, Arruda EM, Faulkner JA, Brooks SV, Larkin LM (2010) Reactive oxygen species on bone mineral density and mechanics in $\mathrm{Cu}, \mathrm{Zn}$ superoxide dismutase (Sod1) knockout mice. Biochem Biophys Res Commun 403(1):149-153

Sözen T, Özışık L, Başaran N (2017) An overview and management of osteoporosis. Eur J Rheumatol 4(1):46-56

Sun YX, Xu AH, Yang Y, Li J (2015) Role of Nrf2 in bone metabolism. J Biomed Sci 22:101

Wang C, Youle RJ (2009) The role of mitochondria in apoptosis. Annu Rev Genet 43:95-118

Yin H, Wang J, Wu M, Ma Y, Wang S, Su Q (2019) Preventive effects of evodiamine on dexamethasone-induced osteoporosis in zebrafish. Biomed Res Int 2019:5859641

Zhang D, Li X, Hu Y, Jiang H, Wu Y, Ding Y, Yu K, He H, Xu J, Sun L, Qian F (2018) Tabersonine attenuates lipopolysaccharide-induced acute lung injury via suppressing TRAF6 ubiquitination. Biochem Pharmacol 154:183-192

\section{Publisher's Note}

Springer Nature remains neutral with regard to jurisdictional claims in published maps and institutional affiliations. 